\title{
The effect of renal diet in association with enalapril or benazepril on proteinuria in dogs with proteinuric chronic kidney disease
}

\author{
A. Zatellii ${ }^{1, *}$, X. Roura ${ }^{2}$, P. D'Ippolito ${ }^{1}$, M. Berlanda ${ }^{3}$ and E. Zini ${ }^{3}, 4,5$ \\ ${ }^{1}$ Medical Consultancy Services, G. Calì Street 60, TBX1424 TàXbiex, Malta \\ ${ }^{2}$ Hospital Clínic Veterinari, Universitat Autònoma de Barcelona, Spain \\ ${ }^{3}$ Department of Animal Medicine, Production and Health, viale dell'Università 16, 35020 Legnaro (PD), University \\ of Padova, Italy \\ ${ }^{4}$ Clinic for Small Animal Internal Medicine, Vetsuisse Faculty, University of Zurich, Winterthurerstrasse 260, 8057 \\ Zurich, Switzerland \\ ${ }^{5}$ Istituto Veterinario di Novara, Strada Provinciale 9, 28060 Granozzo con Monticello (NO), Italy
}

\begin{abstract}
Treating proteinuria in dogs reduces the progression of chronic kidney disease (CKD); renal diets and angiotensinconverting enzyme (ACE)-inhibitors are cornerstones of treatment. Whether different ACE-inhibitors have distinct kidney protective effects is unknown; it is therefore hypothesized that renal diets and enalapril or benazepril have different beneficial effects in proteinuric CKD dogs. Forty-four dogs with proteinuric CKD (IRIS stages 1-4) were enrolled in the study and were fed renal diet for 30 days. Thereafter, they were randomly assigned to one of 2 groups. Dogs in group A ( $\mathrm{n}=22)$ received enalapril $(0.5 \mathrm{mg} / \mathrm{kg}, \mathrm{q} 12 \mathrm{~h})$ and in group B $(\mathrm{n}=22)$ benazepril $(0.5 \mathrm{mg} / \mathrm{kg}, \mathrm{q} 24 \mathrm{~h})$; in both groups, dogs were fed the same renal diet. After randomization, dogs were monitored for 120 days. Body weight and body condition score (BCS), serum concentrations of creatinine, blood urea nitrogen (BUN), albumin and total proteins, and urine protein-to-creatinine (UPC) ratio were compared at different time-points. After 30 days of renal diet, creatinine, BUN and UPC ratio decreased significantly $(\mathrm{p}<0.0001)$. Compared to randomization, body weight, BCS, albumin, total proteins, creatinine and BUN did not vary during follow-up in the 44 dogs and differences between group A and B were not observed. However, the UPC ratio of group A at day 60, 90 and 150 was significantly lower than in group B and compared to randomization $(p<0.05)$. In group B it did not vary overtime. It is concluded that the renal diet is beneficial to decrease creatinine, BUN and UPC ratio in proteinuric CKD dogs. Enalapril further ameliorates proteinuria if administered along with renal diet.
\end{abstract}

Keywords: ACE-I, CKD, Diet, Proteinuria.

\section{Introduction}

Proteinuria has a high prevalence in dogs with chronic kidney disease $(\mathrm{CKD})$ and several studies have shown its role in promoting the progression of renal disorders (Jacob et al., 2002; D'Amico and Bazzi, 2003). In endemic areas for vector-borne diseases, such as leishmaniosis, the prevalence of proteinuric CKD has been reported to vary between $30.2 \%$ and $52.7 \%$ (Cortadellas et al., 2006). Among dogs at risk for developing proteinuric $\mathrm{CKD}$, other than those living or having lived in endemic areas for vector-borne disease, there are also some breeds that are genetically predisposed to develop proteinuric glomerulonephritis (Chew et al., 2011; Harley and Langston, 2012). Early identification and treatment of proteinuria is important in dogs, not only because of the high prevalence of proteinuric $\mathrm{CKD}$, but also because its management allows to slow the progression of CKD, thus leading to decrease the risk of uremic crisis, and kidney-related mortality (Jacob et al., 2002; Jacob et al., 2005; Brown et al., 2013). Angiotensin-converting enzyme (ACE)-inhibitors and dietary intervention are the major cornerstones of treatment in proteinuric CKD dogs; they have been shown to minimize clinical signs of uremia and, at least for the latter, to maintain optimal body conditions (Grauer et al., 2000; Tenhündfeld et al., 2009; Brown et al., 2013; Cortadellas et al., 2014). In order to limit the accumulation of nitrogenous waste products and to slow the progression of CKD, dietary protein restriction has been recommended for CKD dogs in International Renal Interest Society (IRIS) stages 1 with urine protein-to-creatinine ratio (UPC) $>0.5$ (IRIS substage P) and in any case in IRIS stages 2, 3 and 4 (Jacob et al., 2002; Brown et al., 2013; Cortadellas et al., 2014; IRIS Guidelines). Nonetheless, in some dogs, antiproteinuric treatments (renal diet administered along with ACE-inhibitors) do not provide substantial reduction of proteinuria (Brown et al., 2013; Bugbee et al., 2014). Previously published study (Cortadellas et al., 2014) demonstrated a doubtful antiproteinuric efficacy of some ACE-inhibitors. Whether different ACE-inhibitors have distinct antiproteinuric and kidney protective effects is unknown; it is possible that renal diets and enalapril or 
benazepril provide different advantages in proteinuric CKD dogs. Moreover, in dogs with severe proteinuric glomerulonephritis (unresponsive to anti-proteinuric treatment) renal diets may not adequately meet protein requirements, thus leading to malnutrition and hypoalbuminemia, both frequent in dogs with proteinuric CKD. Furthermore, malnutrition and hypoalbuminemia have been associated with increased morbidity and risk of mortality (Remillard et al., 2001; Cave, 2010; Parker and Freeman, 2011).

In light of these premises, proteinuric CKD dogs were included to investigate the short-term effect of renal diet given alone and the medium-term effect of the same renal diet combined with the administration of the ACE-inhibitors enalapril or benazepril; their efficacy on body weight and BCS, on serum concentrations of creatinine, blood urea nitrogen (BUN), albumin and total proteins, and on the UPC ratio was assessed.

\section{Animals and inclusion criteria}

\section{Materials and Methods}

Dogs of any age and sexual status were considered for the present study if affected by naturally occurring CKD in IRIS stages 1, 2, 3 and 4 and if proteinuria was documented (UPC ratio $>0.5$; IRIS substage P) (IRIS Guidelines). The database at admission consisted of history, physical examination including body weight and body condition score (BCS; based on a 1 to 5 scale with 3 being optimal) (Thatcher et al., 2010), hematologic and serum biochemical profile, urinalysis, UPC ratio, immunofluorescence antibody titer (IFAT) for Leishmania infantum, Ehrlichia canis and Anaplasma phagocytophilum, and non-invasive determination of systolic arterial pressure.

Dogs presenting inflammatory diseases of the genitourinary tract, cardiac disease, neoplasia or endocrinopathies, severe hypertension $(\geq 180 \mathrm{mmHg})$ (IRIS Guidelines) or anemia with hematocrit $<25 \%$ and/or hemoglobin $<10.0 \mathrm{~g} / \mathrm{dL}$, and seropositive for Leishmania infantum, Ehrlichia canis or Anaplasma phagocytophilum with associated clinical or laboratory signs other than UPC ratio $>0.5$ were excluded. Dogs that had received ACE-inhibitors, angiotensin II receptor blockers, or corticosteroids during the 12 weeks previous to admission were also excluded. Furthermore, to be enrolled, dogs had to show stable renal function, as defined by serum creatinine concentrations that did not increase or decreased by more than $15 \%$ within 4 weeks from admission; a maximum variation of $0.4 \mathrm{mg} / \mathrm{dL}$ have been considered indicative of stable renal function (Jacob et al., 2002; IRIS Guidelines). Dogs were recruited if an informed consent form to participate in the study was signed by the owners.

\section{Study design}

Following admission, all dogs initially included were fed a renal diet (Prescription Diet Canine k/D, Hill's Pet Nutrition, Topeka, KS) for 30 days. After
30 days, dogs were re-evaluated and allocated to one of the two following groups: group A receiving oral enalapril (Enacard, Merial, Milan, Italy) at $0.5 \mathrm{mg} / \mathrm{kg}$, q12h, plus renal diet, and group B receiving oral benazepril (Fortekor, Novartis Animal Health, Milan, Italy) at $0.5 \mathrm{mg} / \mathrm{kg}, \mathrm{q} 24 \mathrm{~h}$, plus the same renal diet. Randomization was achieved with a software (MedCalc $\AA$, Version 11.3.0.0 (http://www. medcalc.com/)). Either enalapril or benazepril were administered using the recommended dosages of their commercial claims. At the time of the study, both drugs were registered for use in proteinuric CKD dogs in the country where the study was performed (Italy). Every dog included in the study was examined at day 0 (admission), at day 30 (randomization) and at days 45, 60, 90 and 150. The list of exams performed during each evaluation is reported in Table 1 . The listed exams were used to evaluate the effect of treatment and to rule-out laboratory abnormalities caused by one of the infectious diseases stated above.

\section{Blood samples}

Venous blood samples $(5.0 \mathrm{~mL}$ each) were collected in overnight fasted dogs and every sample was placed in tubes containing K3-EDTA $(1.5 \mathrm{~mL})$ and in tubes without anticoagulant $(3.5 \mathrm{~mL})$. Serum was obtained within 30 minutes from collection and stored along with samples in K3-EDTA tubes at $4^{\circ} \mathrm{C}$. Hematologic and serum biochemical profiles were determined within 24 hours with standard methods (BC-2800Vet, MINDRAY, Mindray Co., Shenzhen, China; Cobas

Table 1. Time schedule and exams performed at each evaluation in all dogs.

\begin{tabular}{lcccccc}
\hline Exams & \multicolumn{6}{c}{ Time point (days) } \\
\cline { 2 - 7 } & $\mathrm{O}$ & 30 & 45 & 60 & 90 & 150 \\
\hline Body weight & $\mathrm{X}$ & $\mathrm{X}$ & & $\mathrm{X}$ & $\mathrm{X}$ & $\mathrm{X}$ \\
Body condition score & $\mathrm{X}$ & $\mathrm{X}$ & & $\mathrm{X}$ & $\mathrm{X}$ & $\mathrm{X}$ \\
Systolic arterial pressure & $\mathrm{X}$ & $\mathrm{X}$ & & $\mathrm{X}$ & $\mathrm{X}$ & $\mathrm{X}$ \\
Hematology* $^{*}$ & $\mathrm{X}$ & & & & $\mathrm{X}$ & $\mathrm{X}$ \\
Biochemical profile 1** $^{*}$ & $\mathrm{X}$ & & & & $\mathrm{X}$ & $\mathrm{X}$ \\
Biochemical profile 2*** $^{* *}$ & & $\mathrm{X}$ & $\mathrm{X}$ & $\mathrm{X}$ & & \\
Serology $^{\dagger}$ & $\mathrm{X}$ & & & & & $\mathrm{X}$ \\
Urinalysis $^{\ddagger}$ & $\mathrm{X}$ & $\mathrm{X}$ & $\mathrm{X}$ & $\mathrm{X}$ & $\mathrm{X}$ & $\mathrm{X}$ \\
\hline
\end{tabular}

*: Including erythrocyte, leukocytes and platelet counts, hematocrit, hemoglobin, **: Including creatinine, BUN, alanine aminotransferase, alkaline phosphatase, bilirubin, cholesterol, albumin, total proteins, albumin to globulin ratio, serum electrophoresis, ionized and total calcium, phosphorus, sodium, potassium, and chloride, $* * *$ : Including creatinine, BUN, albumin, total proteins, albumin to globulin ratio, ionized and total calcium, phosphorus, sodium, potassium, chloride, ${ }^{\dagger}$ Including IFAT for Leishmania infantum, Ehrlichia canis and Anaplasma phagocytophilum, ${ }^{*}$ : Including chemical-physical and sediment examination, and UPC ratio 
Mira, Roche Diagnostic, Basel, Switzerland). Regarding the biochemical profile, based on the reference range for serum albumin at our laboratory $(2.8-3.8 \mathrm{~g} / \mathrm{dL})$, severe hypoalbuminemia was arbitrarily considered if the concentration was $<2.0 \mathrm{~g} / \mathrm{dL}$. Dogs with severe hypoalbuminemia received oral acetylsalicylic acid at $2.0 \mathrm{mg} / \mathrm{kg}, \mathrm{q} 24 \mathrm{~h}$, to prevent thrombosis (Brown et al., 2013; Dudley et al., 2013).

\section{Urine samples}

To collect urines, ultrasound-guided cystocentesis were performed in every dog using a $5.0 \mathrm{~mL}$ syringe connected to a 23-gauge needle. Before analysis, urine samples were placed in a $10.0 \mathrm{~mL}$, sterile, evacuated collection tube. Urines were stored at room temperature and examined within 4 hours from collection. Urine sediment was obtained by centrifugation (10 minutes at $900 \times \mathrm{g}$ ) of $5.0 \mathrm{~mL}$ of urine, followed by removal of $4.5 \mathrm{~mL}$ of supernatant, and resuspension of the remaining $0.5 \mathrm{~mL}$ of urine. A sample of $12 \mu \mathrm{L}$ of the resuspended sediment was microscopically assessed. Erythrocytes and leukocytes were expressed as mean number of cells/10 high-power fields (hpf, 40× magnification). Urine sediment with bacteriuria, and/or $>5$ erythrocytes/ hpf or leukocytes/hpf, was considered indicative of inflammation (active sediment) and excluded for the UPC ratio measurement (Beatrice et al., 2010). The supernatant was transferred into separate tubes and stored at $-20^{\circ} \mathrm{C}$ to determine urine protein and creatinine concentrations within 7 days. To calculate the UPC ratio, protein concentration $(\mathrm{mg} / \mathrm{dL})$ was measured with pyrogallol red and creatinine $(\mathrm{mg} / \mathrm{dL})$ using the Jaffé method (Real Time Diagnostic System); measurements were performed with an automated spectrophotometer (Cobas Mira, Roche Diagnostic, Basel, Switzerland). Based on a previous study (Rossi et al., 2012), according with laboratory standard operating procedures (SOP), urine samples were manually diluted 1 to 20 with distilled water to fit linearity of the method. Occasionally, particularly concentrated urine samples were further diluted to 1:100 to fit the linearity of the method. Quality control was performed before any work session with two levels (normal and high) of control serums (Normal Control Serum and Pathological Control Serum) for creatinine. For urinary protein a specific control with bovine albumin $(100 \mathrm{mg} / \mathrm{dL})$ was used.

\section{Statistical analysis}

To study the effect of the renal diet on serum concentrations of creatinine and BUN, and on the UPC ratio, values were compared between admission and after 30 days with the Wilcoxon matched pairs test. To verify whether characteristics of the two groups were similar at randomization (i.e. after 30 days of renal diet and before introduction of ACE-inhibitors), age, body weight, serum concentrations of albumin, total proteins, creatinine and BUN, and the UPC ratio were compared with the Mann-Whitney test followed by Bonferroni post hoc test. Sex distribution and BCS were compared between groups with Fisher's exact test. To study the effect of enalapril or benazepril administered along with the renal diet, on serum concentrations of albumin, total proteins, creatinine and BUN, and on the UPC ratio data were analyzed using an unequally spaced repeated measures model (Littell et al., 1998), assuming as covariance structure a spatial power law on the repeated individual values at different times (day 30, 45, 60, 90 and 150) with baseline values as covariates. The model included the fixed effects of treatment (group A vs. B), time and their interaction; the dog was the repeated random factor. For statistical differences showed by time and time $\times$ treatment effects, all pairwise contrasts were also calculated and corrected with Bonferroni post hoc test. Normality of residuals was evaluated by ShapiroWilk test and values $\geq 0.9$ were considered as normal. Independence of residuals was graphically checked. Statistical significance was considered for $p<0.05$. Data were analyzed with softwares (GraphPad QuickCalcs calculator (2002-2005) by GraphPad Software, San Diego, CA; SAS 9.3, SAS Institute, Cary, NC).

\section{Animals}

\section{Results}

Forty-four dogs were included in the study. Median age at admission was 5 years (range: 1-19 years), median body weight was $26 \mathrm{~kg}$ (range: $3.8-69$ ), and the median BCS was 3 (range: 2-4). Twenty-one dogs were intact males, 13 were spayed females, 6 were intact females and 4 were castrated males. Eight dogs were cross-breed and 36 were pure-breed, including 9 Boxers, 4 Yorkshire Terriers, 3 Cocker Spaniels and Golden Retrievers, 2 Bernese Mountain dogs and Rottweilers and, one each of American Staffordshire, Beagle, Bolognese, Italian Bracco, Bull Terrier, Czechoslovakian Wolfdog, Dachshund, Fox Terrier, German Shepherd, Irish Setter, Scottish Terrier, Samoyed and Weimaraner.

\section{Effects of renal diet}

After 30 days of renal diet, serum creatinine and BUN, and UPC ratio decreased (Fig. 1). In particular, median creatinine at admission was $2.4 \mathrm{mg} / \mathrm{dL}$ (range: 1.0-9.1) and after 30 days it was $2.2 \mathrm{mg} / \mathrm{dL}$ (range: $0.6-7.5$ ) $(\mathrm{p}<0.0001)$; creatinine decreased in 38 dogs $(86.4 \%)$ and increased in the remaining $6(13.6 \%)$. Median BUN at admission was $56 \mathrm{mg} / \mathrm{dL}$ (range: 18-148) and after 30 days it was $47 \mathrm{mg} / \mathrm{dL}$ (range: $10-108)(\mathrm{p}<0.0001)$; BUN decreased in 41 dogs (93.2\%) and increased in the remaining $3(6.8 \%)$. Median UPC ratio at admission was 2.9 (range: $0.8-19.8$ ) and after 30 days it was 2.2 (range: $0.6-14.4)(\mathrm{p}<0.0001)$; UPC ratio decreased in 42 dogs $(95.5 \%)$ and increased in the remaining $2(4.5 \%)$. Effects of enalapril or benazepril associated with renal diet

Randomization performed after 30 days of renal diet and before introduction of ACE-inhibitors showed 


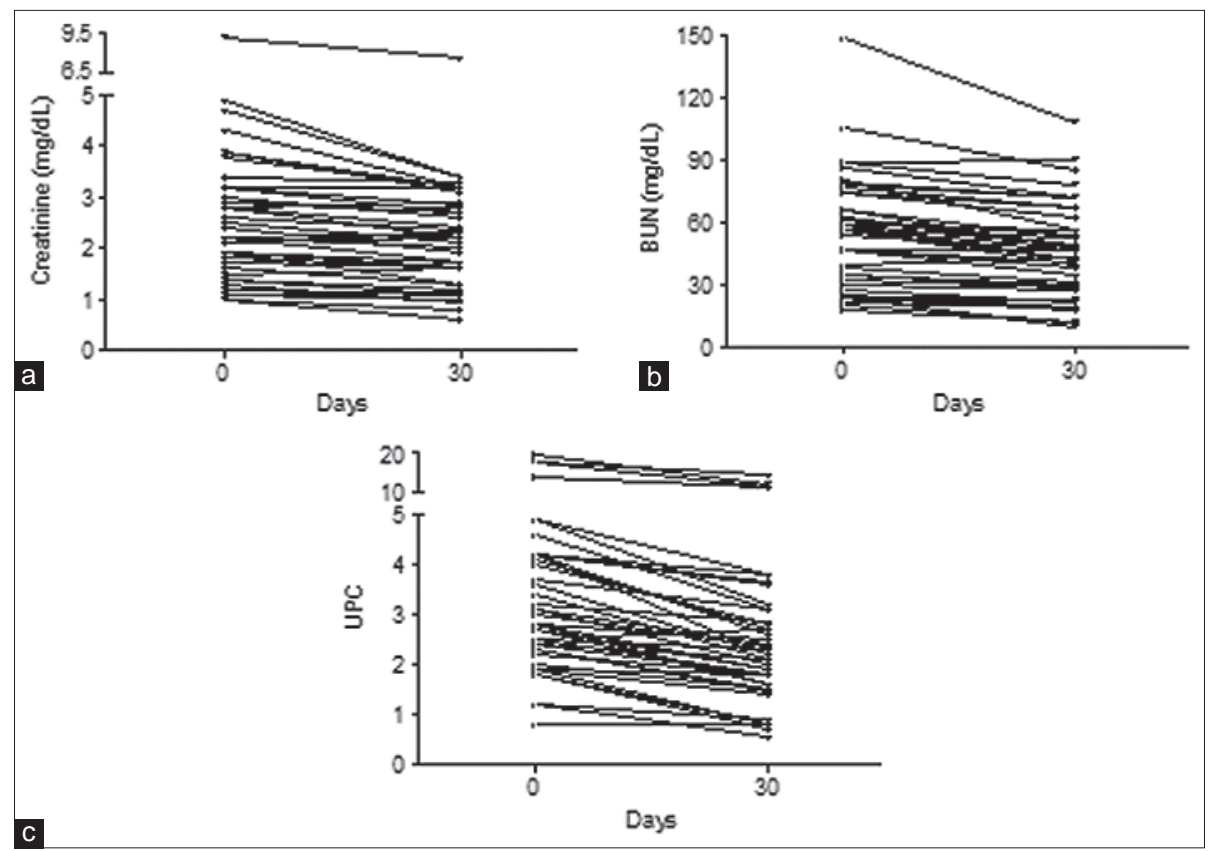

Fig. 1. Serum concentrations of creatinine (a) and BUN (b), and UPC ratio (c) in all dogs before any treatment (day 0 ) and after one month of renal diet (day 30 ).

that age, body weight, BCS, serum concentrations of albumin, total proteins, creatinine and $\mathrm{BUN}$, and the UPC ratio did not differ between dogs allocated to group A or B (Table 2).

Twenty-two dogs were initially included into group A and received enalapril. Twenty-two were included into group B and received benazepril. Both groups were fed the same renal diet. In group A, 3 of the 22 dogs (13.6\%) died during the study period; 2 of them died between 60 and 90 days following the start of the study and 1 between 90 and 150 days. In the group B, 2 of the 22 dogs (9.1\%) died, 1 between 60 and 90 days and 1 between 90 and 150 days following the start of the study. In all 5 dogs, death was due to the progressive worsening of CKD; of the same dogs, after 30 days of renal diet, 5 had a decrease of creatinine, 4 of BUN and 5 of the UPC ratio. Furthermore, 3 dogs (13.6\%) in group B were missed at follow-up visits, 2 between 60 and 90 days and 1 between 90 and 150 days after the start of the study.

Serum concentrations of albumin, total proteins, creatinine and BUN, and UPC ratio of dogs in groups A and $\mathrm{B}$ at randomization and during follow-up are reported in Table 3. Serum concentrations of albumin, total proteins, creatinine and BUN did not vary during the follow-up period to any significant degree in the 44 dogs grouped together, as compared to randomization (day 30). Furthermore, for the same parameters, differences between groups A and B were not observed during treatment. Dogs in group A had significantly lower UPC ratio at day $60(\mathrm{p}<0.05)$, day $90(\mathrm{p}<0.01)$
Table 2. Comparison of variables used to assess adequacy of matching of dog groups. Values are reported as median and range or as number of dogs and frequency.

\begin{tabular}{|c|c|c|c|}
\hline Variable & Group A & Group B & p-value \\
\hline Age (years) & $6(2-12)$ & $4(1-19)$ & 0.916 \\
\hline Sex & & & 1.000 \\
\hline Female & $3(13.6 \%)$ & $3(13.6 \%)$ & \\
\hline Spayed female & $7(31.9 \%)$ & $6(27.3 \%)$ & \\
\hline Male & $9(40.9 \%)$ & $12(54.5 \%)$ & \\
\hline Castrated male & $3(13.6 \%)$ & $1(4.6 \%)$ & \\
\hline $\begin{array}{l}\text { Body } \\
\text { weight (kg) }\end{array}$ & $25.9(4.2-57)$ & $27(3.8-69)$ & 0.916 \\
\hline BCS & & & 1.000 \\
\hline 2 & $6(27.3 \%)$ & $8(36.4 \%)$ & \\
\hline 3 & $15(68.2 \%)$ & $12(54.5 \%)$ & \\
\hline 4 & $1(4.5 \%)$ & $2(9.1 \%)$ & \\
\hline $\operatorname{Albumin}(\mathrm{g} / \mathrm{dL})$ & $2.7(1.7-3.5)$ & $2.9(1.6-4.0)$ & $0.350 *$ \\
\hline $\begin{array}{l}\text { Total proteins } \\
(\mathrm{g} / \mathrm{dL})\end{array}$ & $5.7(4.8-7.0)$ & $6.4(4.9-8.5)$ & $0.084 *$ \\
\hline $\begin{array}{l}\text { Creatinine } \\
(\mathrm{mg} / \mathrm{dL})\end{array}$ & $1.8(0.6-3.4)$ & $2.3(1.0-3.3)$ & 0.117 \\
\hline BUN (mg/dL) & $42(12-72)$ & $49(10-108)$ & 0.110 \\
\hline UPC ratio & $2.3(0.7-11.5)$ & $2.0(0.6-14.4)$ & 0.404 \\
\hline
\end{tabular}

*: $p$-value corrected with Bonferroni's post hoc test

and day $150(\mathrm{p}<0.05)$ as compared to randomization (Table 3), whereas dogs in group B had not different UPC 
Table 3. Serum concentrations of albumin, total proteins, creatinine and BUN, and UPC ratio of dogs in groups A and B at randomization (30 days) and follow-up evaluations. Median and range are reported.

\begin{tabular}{|c|c|c|c|c|c|}
\hline \multirow[t]{2}{*}{ Variable } & \multicolumn{5}{|c|}{ Time point (days) } \\
\hline & 30 & 45 & 60 & 90 & 150 \\
\hline \multicolumn{6}{|c|}{ Albumin (g/dL) } \\
\hline Group A & $2.6(1.7-3.5)$ & $2.7(1.6-3.4)$ & $2.6(1.9-3.4)$ & $2.7(1.9-3.5)$ & $2.7(2.1-3.4)$ \\
\hline Group B & $2.9(1.6-4.0)$ & $2.8(1.7-3.6)$ & $2.8(1.8-3.6)$ & $2.8(2.0-3.3)$ & $2.8(1.8-3.1)$ \\
\hline \multicolumn{6}{|c|}{ Total proteins $(\mathrm{g} / \mathrm{dL})$} \\
\hline Group A & $5.7(4.8-7.0)$ & $5.8(4.8-6.7)$ & $5.8(5.0-6.8)$ & $5.9(5.2-6.8)$ & $5.8(5.1-7.0)$ \\
\hline Group B & $6.4(4.9-8.5)$ & $6.3(4.8-7.9)$ & $6.3(5.0-7.7)$ & $6.0(5.2-7.7)$ & $6.2(5.1-6.9)$ \\
\hline \multicolumn{6}{|c|}{ Creatinine (mg/dL) } \\
\hline Group A & $1.8(0.6-3.4)$ & $1.8(0.7-3.7)$ & $1.6(0.8-3.9)$ & $1.6(0.9-4.3)$ & $1.5(0.9-3.4)$ \\
\hline Group B & $2.3(1.0-3.3)$ & $2.4(1.0-5.0)$ & $2.3(0.8-5.5)$ & $2.3(0.9-7.5)$ & $2.4(0.9-5.0)$ \\
\hline \multicolumn{6}{|c|}{ BUN (mg/dL) } \\
\hline Group A & $42(12-72)$ & $42(17-66)$ & $40(13-89)$ & $37(15-89)$ & $36(16-67)$ \\
\hline Group B & $49(10-108)$ & $49(19-112)$ & $50(16-143)$ & $50(20-100)$ & $50(20-85)$ \\
\hline \multicolumn{6}{|l|}{ UPC } \\
\hline Group A & $2.3(0.7-11.5)$ & $2.3(0.8-8.0)$ & $1.6(0.4-5.6)^{*}$ & $1.2(0.3-8.9)^{\dagger}$ & $1.2(0.5-4.5)^{*}$ \\
\hline Group B & $2.0(0.6-14.4)$ & $2.2(0.6-9.2)$ & $2.2(0.5-9.8)$ & $1.8(0.6-9.0)$ & $1.9(0.6-9.2)$ \\
\hline
\end{tabular}

Significant differences between follow-up evaluations and randomization within groups: ${ }^{*}: p<0.05 ;{ }^{\dagger}: p<0.01$

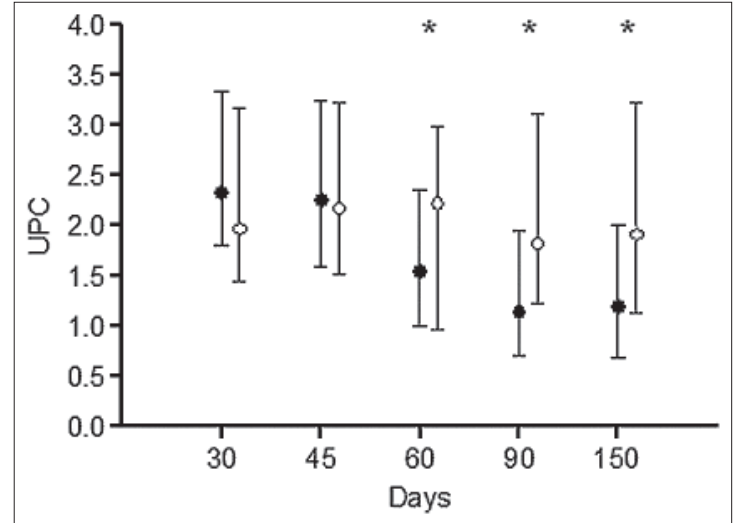

Fig. 2. UPC ratio in dogs receiving enalapril and renal diet (group A, black dots) or benazepril and renal diet (group B, white dots) at randomization (day 30) and follow-up. Median values and interquartile ranges are shown. Significant differences between group A and B are depicted with asterisks $(\mathrm{p}<0.05)$.

ratio at each time point during follow-up compared to randomization. Dogs in group A had significantly lower UPC ratio than dogs in group B at day $60(\mathrm{p}<0.05), 90$ $(p<0.05)$ and $150(p<0.05)$ (Fig. 2).

\section{Discussion}

Because persistent renal proteinuria in dogs has been associated with a poorer prognosis (Harley and Langston, 2012) its treatment is recommended in order to improve quality of life and survival time. For this purpose, the potential beneficial effects of renal diet and of the ACE-inhibitors enalapril and benazepril in dogs with proteinuric CKD were assessed in the present study. With regard to dietary management, we substantiated the beneficial effect of using renal diet in proteinuric CKD dogs. Indeed, during the first 30 days of the trial, the enrolled dogs were managed with the renal diet but did not receive any medication and a significant improvement of serum concentrations of creatinine and BUN, as well as the UPC ratio was observed compared to baseline. Main characteristics of the selected diet, which are the key points of the dietary management (Roudebush et al., 2010; Polzin, 2013) are protein restriction with high biological value, phosphorus and sodium reduction and supplementation with $\omega-3$ polyunsaturated fatty acids (PUFAs). Among them, content and quality of proteins as well as phosphorus and sodium restriction seem to be the most important aspects to consider in proteinuric CKD dogs, whereas the effect of PUFAs is yet not fully known and deserves further investigation (Roudebush et al., 2010; Brown et al., 2013).

For the present trial, the ACE-inhibitors enalapril and benazepril were administered using the recommended dosages of their commercial claims. The group treated with enalapril showed a significant reduction of the UPC ratio after 30 days of treatment (60 days from the beginning of the study); however, the group treated with benazepril did not show ameliorations of the UPC ratio, at any time. One recent study showed 
that benazepril might not be effective in reducing renal proteinuria in dogs (Cortadellas et al., 2014). However, as proposed by the same authors, the lack of efficacy of benazepril in their investigation might have been due to the drug dosage used (Cortadellas et al., 2014). Indeed, based on studies in humans, increasing the dosage of ACE-inhibitors can improve the antiproteinuric effect, possibly suggesting that higher dosages of benazepril might also be beneficial to decrease proteinuria in CKD dogs (Weinberg et al., 2001; Tylicki et al., 2012). On the other hand, another investigation in dogs showed a reduction of the UPC ratio associated with the administration of benazepril (Tenhündfeld et al., 2009). However, comparison between our results and those of Tenhündfeld et al. (2009) is difficult because they had three different groups of only 10 dogs, treated with placebo, benazepril and benazepril plus heparin, which were not homogeneous for UPC ratio at the time treatment was started. Furthermore, they observed a beneficial effect on UPC ratio only by day 180 for dogs in the benazepril group and by day 90 for dogs in the benazepril plus heparin group. Therefore, if an effect of benazepril exists on proteinuria, it would likely be delayed compared to that achieved with enalapril, questioning the real benefit of benazepril to treat proteinuric CKD dogs based on the current recommended dosages.

In the present study, the significant reduction of UPC ratio in the group treated with enalapril was evident by 30 days of therapy, whereas no significant amelioration was observed if tested after only 15 days of treatment with the ACE-inhibitor. This result may provide useful information for the monitoring of proteinuric CKD dogs in clinical practice. To assess the potential favorable effect of enalapril to control proteinuria in dogs, the UPC ratio should be evaluated after 30 days of treatment; therefore, absence of improvement at 15 days should not be regarded as indicative of lack of efficacy.

During the study period, none of the dogs developed severe hypertension and thus needed to receive amlodipine or other anti-hypertensive treatments. The fact that blood pressure did not increase throughout the study may be due to the limited duration of the trial, or to the administration of ACE-inhibitors.

Some limitations of the study need to be mentioned, including the relative small sample size, the lack of blinding and of renal biopsies. The risk associated with lack of masking was minimized by randomization and by excluding statistical differences between the groups at the time the ACE-inhibitors were started. Absence of renal histology did not allow excluding that, in some dogs, proteinuria was primarily due to tubular or tubulointerstitial damage, rather than being of glomerular origin. However, if this was the case, it is likely that dogs with tubular or tubulointerstitial damage were evenly distributed between groups. In addition, dogs with severe hypoalbuminemia $(<2 \mathrm{~g} / \mathrm{dL})$ received oral acetylsalicylic acid. As hypothesized by some authors (Grauer et al., 2000; Cortadellas et al., 2014), low dosages of acetylsalicylic acid have a beneficial effect on proteinuria in dogs with glomerulonephritis and, therefore, its use might have partly biased the results of the study. Nonetheless, the potential beneficial effect of acetylsalicylic acid was likely similar between groups as the frequency of dogs with severe hypoalbuminemia that received it did not differ.

In conclusion, the administration of renal diet ameliorates serum creatinine, BUN and UPC ratio, and adding enalapril decreases the UPC ratio further in dogs with proteinuric CKD. Using benazepril, at the present dosage and for 120 days, was not beneficial compared with enalapril. Future studies are needed to verify the effect of benazepril over longer periods or at higher dosages in dogs with proteinuric CKD, or that of angiotensin II receptor blockers or PUFAs, administered along with renal diet.

\section{Conflict of interest}

The Authors declares that there is no conflict of interest.

\section{Acknowledgment}

The study is partly supported by Merial Italy. The authors are grateful to Dr. Barbara Contiero for her statistical advice.

\section{References}

Beatrice, L., Nizi, F., Callegari, D., Paltrinieri, S., Zini, E., D'Ippolito, P. and Zatelli, A. 2010. Comparison of urine protein-to-creatinine ratio in urine samples collected by cystocentesis versus free catch in dogs. J. Am. Vet. Med. Assoc. 236, 1221-1224.

Brown, S., Elliott, J., Francey, T., Francey, T., Polzin, D. and Vaden, S. 2013. Consensus recommendations for standard therapy of glomerular disease in dogs. J. Vet. Intern. Med. 27(Suppl 1), S27-43.

Bugbee, A.C., Coleman, A.E., Wang, A., Woolcock, A.D. and Brown, S.A. 2014. Telmisartan treatment of refractory proteinuria in a dog. J. Vet. Intern. Med. 28, 1871-1874.

Cave, N. 2010. Immunology and nutrition. In: Ettinger SJ, Feldman EC, eds. Textbook of veterinary internal medicine. $7^{\text {th }}$ ed. St. Louis: Saunders Elsevier, pp: 638-642.

Chew, D.J., DiBartola, S.P. and Schenck, P.A. 2011. Familial renal diseases of dogs and cats. In: Chew DJ, DiBartola SP, Schenck PA, eds. Canine and feline nephrology and urology $2^{\text {nd }}$ ed. St. Louis, Missouri, Elsevier Saunders, pp: 197-211.

Cortadellas, O., Fernández del Palacio, M.J., Bayón, A., Albert, A. and Talavera, J. 2006. Systemic hypertension in dogs with leishmaniasis: prevalence and clinical consequences. J. Vet. Intern. Med. 20, 941-947. 
Cortadellas, O., Talavera, J. and Fernández del Palacio, M.J. 2014. Evaluation of the effects of a therapeutic renal diet to control proteinuria in proteinuric non-azotemic dogs treated with benazepril. J. Vet. Intern. Med. 28, 30-37.

D’Amico, G. and Bazzi, C. 2003. Pathophysiology of proteinuria. Kidney Int. 63, 809-825.

Dudley, A., Thomason, J., Fritz, S., Grady, J., Stokes, J., Wills, R., Pinchuk, L., Mackin, A. and Lunsford, K. 2013. Cyclooxygenase expression and platelet function in healthy dogs receiving lowdose aspirin. J. Vet. Intern. Med. 27, 141-149.

Grauer, G.F., Greco, D.S., Getzy, D.M., Cowgill, L.D., Vaden, S.L., Chew, D.J., Polzin, D.J. and Barsanti, J.A. 2000. Effects of enalapril versus placebo as a treatment for canine Idiopathic glomerulonephritis. J. Vet. Intern. Med. 14, 526-533.

Harley, L. and Langston, C. 2012. Proteinuria in dogs and cats. Can. Vet. J. 53, 631-638.

IRIS Guidelines. International Renal Interest Society. Available at: http://www.iris-kidney.com/ guidelines.

Jacob, F., Polzin, D.J., Osborne, C.A., Allen, T.A., Kirk, C.A., Neaton, J.D., Lekcharoensuk, C. and Swanson, L.L. 2002. Clinical evaluation of dietary modification for treatment of spontaneous chronic renal failure in dogs. J. Am. Vet. Med. Assoc. 220, 1163-1170.

Jacob, F., Polzin, D.J., Osborne, C.A., Neaton, J.D., Kirk, C.A., Allen, T.A. and Swanson, L.L. 2005. Evaluation of the association between initial proteinuria and morbidity rate or death in dogs with naturally occurring chronic renal failure. J. Am. Vet. Med. Assoc. 226, 393-400.

Littell, R.C., Henry, P.R. and Ammerman, C.B. 1998. Statistical analysis of repeated measures data using SAS procedures. J. Anim. Sci. 76, 1216-1231.
Parker, V.J. and Freeman, L.M. 2011. Association between body condition and survival in dogs with acquired chronic kidney disease. J. Vet. Intern. Med. 25, 1306-1311.

Polzin, D.J. 2013. Evidence-based step-wise approach to managing chronic kidney disease in dogs and cats. J. Vet. Emerg. Crit. Care (San Antonio) 23, 205-215.

Remillard, R.L., Darden, D.E., Michel, K.E., Marks, S.L., Buffington, C.A. and Bunnell, P.R. 2001. An investigation of the relationship between caloric intake and outcome in hospitalized dogs. Vet. Ther. 2, 301-310.

Roudebush, P., Polzin, D.J., Adams, L.G., Towell, T.L. and Forrester, S.D. 2010. An evidence-based review of therapies for canine chronic kidney disease. J. Small Anim. Pract. 51, 244-252.

Tenhündfeld, J., Wefstaedt, P. and Nolte, I.J. 2009. A randomized controlled clinical trial of the use of benazepril and heparin for the treatment of chronic kidney disease in dogs. J. Am. Vet. Med. Assoc. 234, 1031-1037.

Thatcher, C.D., Hand, M.S. and Remillard, R.L. 2010. Small animal clinical nutrition: an iterative process. In: Hand, M.S., Thatcher, C.D., Remillard, R.L., Roudebush, P. and Novotny, B.J., eds. Small animal clinical nutrition. $5^{\text {th }}$ ed. Topeka, Kansas: Mark Morris Institute, pp: 3-21.

Tylicki, L., Lizakowski, S. and Rutkowski, B. 2012. Renin-angiotensin-aldosterone system blockade for nephroprotection: current evidence and future directions. J. Nephrol. 25, 900-910.

Weinberg, M., Weinberg, A., Cord, R. and Zappe, D. 2001. The effect of high-dose angiotensin II receptor blockade beyond maximal recommended doses in reducing urinary protein excretion. J. Renin Angiotensin Aldosterone Syst. 2(Suppl 1), 196-198. 